\title{
Integrated waveguide and micro-ring resonator polarizers via integrated 2D graphene oxide films
}

This paper was downloaded from TechRxiv (https://www.techrxiv.org).

\section{LICENSE}

CC BY 4.0

\section{SUBMISSION DATE / POSTED DATE}

29-08-2020 / 09-09-2020

\section{CITATION}

Moss, David (2020): Integrated waveguide and micro-ring resonator polarizers via integrated 2D graphene oxide films. TechRxiv. Preprint. https://doi.org/10.36227/techrxiv.12893324.v1

DOI 


\title{
Integrated waveguide and micro-ring resonator polarizers via integrated 2D graphene oxide films
}

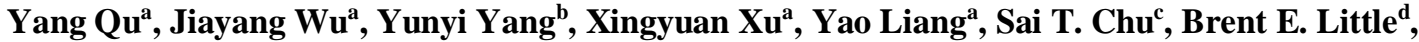 \\ Roberto Morandotti ${ }^{\mathrm{e}}$, Baohua Jia, ${ }^{\mathrm{b}}$ and David J. Moss ${ }^{\mathrm{a}, *}$ \\ ${ }^{a}$ Optical Sciences Centre, Swinburne University of Technology, Hawthorn, VIC 3122, Australia \\ ${ }^{b}$ Centre for Translational Atomaterials, Swinburne University of Technology, Hawthorn, VIC 3122, Australia \\ ${ }^{c}$ City University of Hong Kong, Tat Chee Avenue, Hong Kong, China \\ ${ }^{d} X i$ 'an Institute of Optics and Precision Mechanics Precision Mechanics of CAS, Xi'an, China \\ eINRS -Énergie, Matériaux et Télécommunications, 1650 Boulevard Lionel-Boulet, Varennes, Québec, Canada
}

\begin{abstract}
We experimentally demonstrate integrated waveguide and micro-ring resonator polarizers incorporating two-dimensional layered graphene oxide films, with a high polarization dependent loss of $53.8 \mathrm{~dB}$ and a high polarization extinction ratio of $8.3 \mathrm{~dB}$, respectively.
\end{abstract}

\section{Introduction}

Polarization control is a fundamental requirement in many optical technologies [1-3]. Integrated polarization selective devices based on complementary metal-oxide-semiconductor (CMOS) compatible integrated platforms [4,5] offer competitive advantages of compact footprint, high stability, mass producibility and high scalability as functional building blocks for photonic integrated circuits [2]. Recently, the huge optical anisotropy and broadband response of two dimensional (2D) materials such as graphene and transition metal dichalcogenides have been widely recognized and exploited to implement polarization-selective devices [6-9]. However, none of these demonstrations were based on CMOS compatible platforms. Generally, the integration of 2D materials on CMOS compatible platforms requires layer transfer processes. Despite its widespread implementation, the transfer approach itself is complex, which makes it difficult to achieve precise patterning, as well as flexible placement and large-area continuous coating on integrated devices.

Owing to its ease of preparation as well as the tunability of its material properties [10], graphene oxide (GO) has become a highly promising member of the 2D family. Recently [10], we reported large-area, transfer-free, and high-quality GO film coating on integrated waveguides using a solution-based method with layer-by-layer deposition of GO films. Here, we use these techniques to demonstrate GO-coated integrated waveguide and microring resonator (MRR) polarizers on a CMOS compatible doped silica platform [11]. We achieve highly precise control of the placement, thickness, and length of the GO films coated on integrated photonic devices by using our layer-by-layer GO coating method followed by photolithography and lift-off processes. We measure the performance of the waveguide polarizer for different GO film thicknesses and lengths versus polarization, wavelength, and power, achieving a very high polarization dependent loss (PDL) of $\sim 53.8 \mathrm{~dB}$. For GO-coated integrated MRRs, we achieve an 8.3-dB polarization extinction ratio between the TE and TM resonances. These results confirm the high-performance of integrated polarization selective devices incorporated with GO films.

\section{Device fabrication and experimental results}

Figure 1 (a) shows a schematic of a uniformly GO-coated waveguide polarizer. The waveguides were fabricated from high-index doped silica glass core $(\sim 2 \mu \mathrm{m}$ wide $\times \sim 1.5 \mu \mathrm{m}$ high $)$ surrounded by silica. 1 to 10 layers of GO were uniformly coated on doped silica waveguides. Fig. 1(b) shows the measured Raman spectra of the waveguides without $\mathrm{GO}$ and with 2 layers of GO, confirming the integration of $\mathrm{GO}$ onto the top surface by the presence of the $\mathrm{D}\left(1345 \mathrm{~cm}^{-1}\right)$ and $\mathrm{G}\left(1590 \mathrm{~cm}^{-1}\right)$ peaks of GO. Fig. 1(c) is a microscope image of the integrated MRR patterned with 50 layers of GO. The combination of patterning and deposition control of GO films along with large area coating capability is critical for large-scale integrated devices incorporated with GO.

Figure 2 shows the polarization dependent (TE (in-plane) and TM (out-of-plane)) performance for both the 1.5$\mathrm{cm}$-long uniformly coated waveguides (0-10 layers), left side (i), and the patterned 2-mm-long devices (10-100 layers), right side (ii). Figure 2(a) shows the polarization dependent insertion loss. Figure 2(b) presents the

(a)
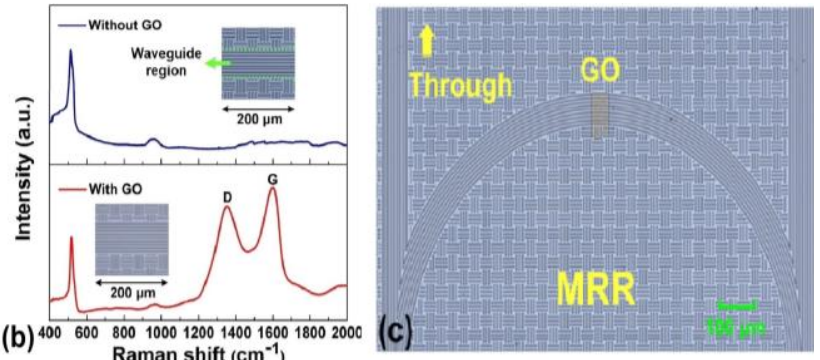

Fig. 1 (a) Schematic illustration of GO-coated integrated waveguide polarizer. (b) Raman spectra of the integrated chip without GO and with 2 layers of GO. Insets show the corresponding microscope images. (c) Microscope images of an MRR patterned with 50 layers of GO 

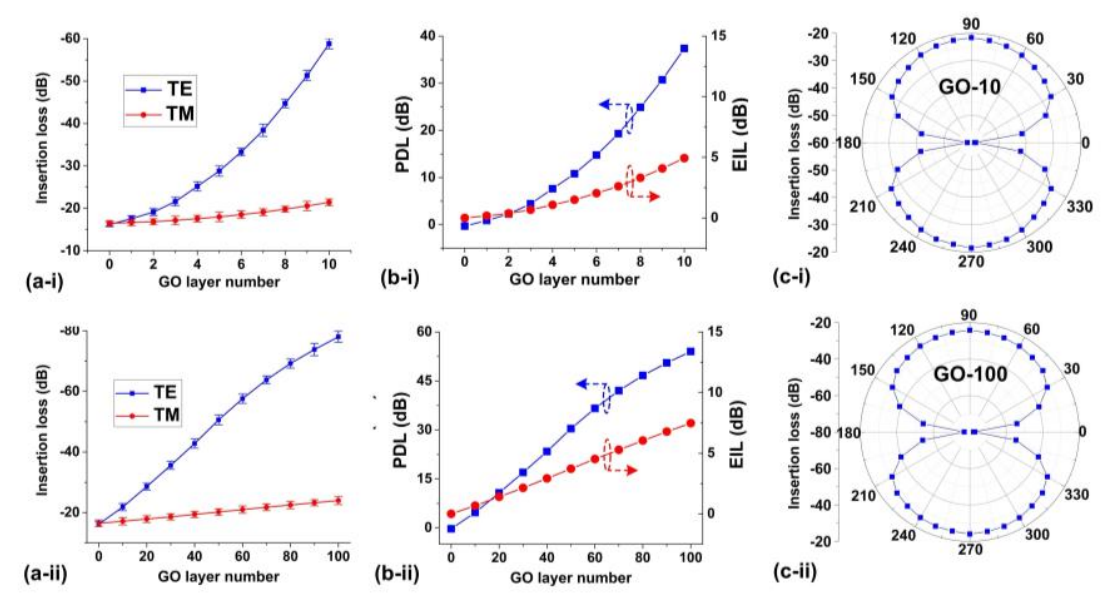

Fig. 2 (a) Measured TE and TM polarized insertion loss. (b) Extracted polarization dependent loss (PDL) and excess insertion loss (EIL). (c) Polar diagrams for the insertion loss. In (a) - (c), (i) shows the results for 1.5 -cm-long uniformly coated waveguides $(0,1,2, \ldots, 10$ layers of $\mathrm{GO})$ and (ii) shows the results for 1.5 -cm-long waveguides with 2 -mm-long patterned $\mathrm{GO}(0,10,20, \ldots, 100$ layers of GO). The input $\mathrm{CW}$ power and wavelength in (a) are $0 \mathrm{dBm}$ and $1550 \mathrm{~nm}$.

polarization dependent loss (PDL), which defined as the ratio of the maximum to minimum insertion losses, and excess insertion loss (EIL), the insertion loss induced by the GO film over the uncoated waveguide. Figures 2(ci) and 2(c-ii) are the polar diagrams for the insertion loss, respectively. The TE insertion loss increases much more strongly than TM with layer number, thus forming the basis for high-performance polarization dependent devices.

The measured TE and TM polarized transmission spectra of the uniformly GO-coated MRR are shown in Figs. 3(a) and (b), respectively, while the transmission spectra of the patterned MRR are shown in Figs. 3(c) and (d), all measured with the same doped silica MRR at a continuous wave power of $\sim 0 \mathrm{dBm}$. For the patterned MRR with 50 layers of GO, a maximum polarization extinction ratio (defined as the difference between the extinction ratios of the TE and TM polarized resonances) of $\sim 8.3 \mathrm{~dB}$ was achieved. For nonlinear applications[12], compared

(a) TE

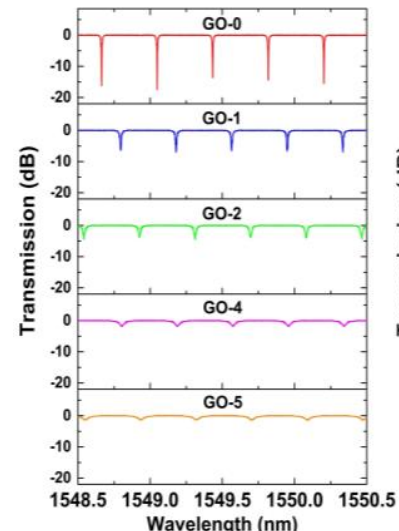

(b) TM

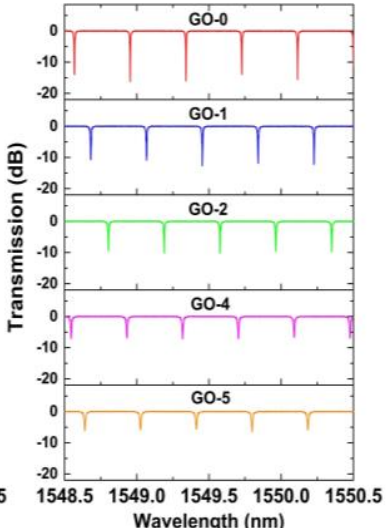

(c) TE

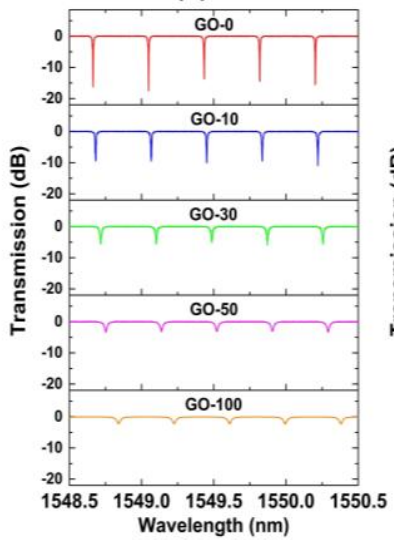

(d) TM

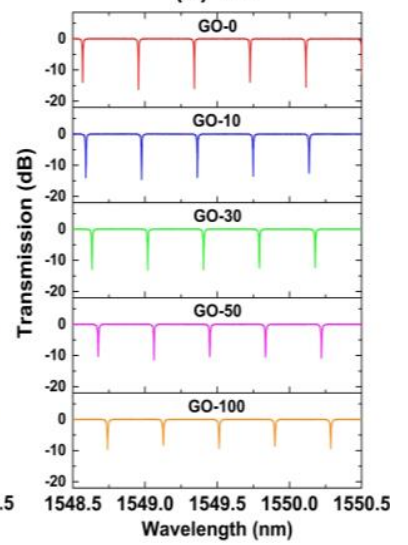

Fig. 3. (a) - (b) Measured TE and TM polarized transmission spectra of the MRR uniformly coated with 0-5 layers of GO. (c)-(d) Measured TE and TM polarized transmission spectra of the MRR patterned with 10-100 layers of GO.

with other 2D materials, $n_{2}$ for GO is lower than graphene but still two orders of magnitude higher than bulk silicon [13,14], which demonstrates the high potential of GO as a new optical material for nonlinear photonic applications. Further, like Si-Ge heterostructures, $[15,16]$ GO may also offer interesting possibilities for both $2^{\text {nd }}$ and $3^{\text {rd }}$ order nonlinear effects courtesy of its complex anisotropic nonlinear optical characteristics.

\section{Conclusion}

Integrated waveguide and MRR polarizers incorporating 2D layered GO films are experimentally demonstrated with a high polarization dependent loss of $53.8 \mathrm{~dB}$ and a high polarization extinction ratio of $8.3 \mathrm{~dB}$, respectively.

\section{References}

[1] Y. Yan et al., "High-capacity millimetre-wave communications with orbital angular momentum multiplexing", Nat. Commun., 5, 4876, 2014.

[2] D. Dai et al., "Passive technologies for future large-scale photonic integrated circuits on silicon: polarization handling, light non-reciprocity and loss reduction", Light Sci. Appl., 1: e1, 2012.

[3] D. Dai et al., "Polarization management for silicon photonic integrated circuits", Laser Photonics Rev., 7, $303,2013$.

[4] D.J. Moss et al., "New CMOS-compatible platforms based on silicon nitride and Hydex for nonlinear optics", Nat. Photonics. 7 (8) 597, 2013.

[5] H. Bao, et.al, "Laser Cavity-Soliton Micro-Combs", Nature Photonics $\underline{13}$ (6) 384-389 (2019). 
[6] Q. Bao et al., "Broadband graphene polarizer" Nat. Photonics, 5, 411, 2011.

[7] de Oliveira et al., "Graphene based waveguide polarizers: in-depth physical analysis and relevant parameters", Sci. Rep.,5(1), 16949, 2015.

[8] H. Lin et al., "Chalcogenide glass-on-graphene photonics" Nat. Photonics, 11, 798, 2017.

[9] X. Zheng et al., "In situ third-order non-linear responses during laser reduction of graphene oxide thin films towards onchip non-linear photonic devices", Adv. Mater., 26, 2699, 2014.

[10] Y. Yang et al., "Enhanced four-wave mixing in waveguides integrated with graphene oxide", APL Photonics, 3, 120803, 2018.

[11] J. Wu et.al., "Graphene oxide waveguide and micro-ring resonator polarizers," Laser Photonics Rev., 13(9), 1900056, 2019.

[12] J.Wu et.al., "Enhanced nonlinear FWM in MRRs integrated with layered GO films", Small 16(16), 1906563 (2020).

[13] Y. Zhang et.al, "Enhanced Kerr nonlinearity and nonlinear figure of merit in silicon nanowires integrated with 2D graphene oxide films", ACS Applied Materials and Interfaces 12 (29) 33094-33103 (2020).

[14] D.J. Moss, et.al, "Dispersion in the anisotropy for optical third harmonic generation in Si and Ge", Optics Letters 14 (1), 57-59 (1989).

[15] M.Sinobad et.al., "Mid-IR octave spanning supercontinuum generation to $8.5 \mu \mathrm{m}$ in Si-Ge waveguides", Optica $\underline{\mathbf{5}}$ (4), 360-366 (2018).

[16] E. Ghahramani, D.J. Moss, and J.E. Sipe, "Second-harmonic generation in odd-period, strained, $(\mathrm{Si}) \mathrm{n}(\mathrm{Ge}) \mathrm{n} / \mathrm{Si}$ superlattices and at $\mathrm{Si} / \mathrm{Ge}$ interfaces", Physical Review Letters, vol. 64, no. 23, pp.2815-2818 (1990). DOI:10.1103/PhysRevLett.64.2815. 